\title{
A Quantitative Targeted Proteomics Approach to Validate Predicted microRNA Targets in C. elegans
}

\author{
Marko Jovanovic ${ }^{1,2,3,12}$, Lukas Reiter ${ }^{1,2,3,10,12}$, Paola Picotti ${ }^{4}$, Vinzenz Lange ${ }^{4,5,11}$, Erica \\ Bogan $^{1,2}$, Benjamin A. Hurschler ${ }^{6}$, Cherie Blenkiron ${ }^{7,8}$, Nicolas J. Lehrbach ${ }^{7,8}$, Xavier C. \\ Ding $^{6}$, Manuel Weiss ${ }^{1,2,3}$, Sabine P. Schrimpf ${ }^{1,3}$, Eric A. Miska ${ }^{7,8}$, Helge Grosshans ${ }^{6}$, Ruedi \\ Aebersold ${ }^{4,5,9, *}$, and Michael O. Hengartner ${ }^{1,3,}{ }^{*}$
}

${ }^{1}$ Institute of Molecular Biology, University of Zurich, Zurich, Switzerland ${ }^{2} \mathrm{PhD}$ Program in Molecular Life Sciences Zurich, Zurich, Switzerland ${ }^{3}$ Quantitative Model Organism Proteomics (Q-MOP), University of Zurich, Zurich, Switzerland ${ }^{4}$ Institute of Molecular Systems Biology, ETH Zurich, Zurich, Switzerland ${ }^{5}$ Competence Center for Systems Physiology and Metabolic Diseases, Zurich, Switzerland ${ }^{6}$ Friedrich Miescher Institute for Biomedical Research (FMI), Basel, Switzerland ${ }^{7}$ Department of Biochemistry, University of Cambridge, Cambridge, CB2 1GA, United Kingdom ${ }^{8}$ Wellcome Trust/Cancer Research UK Gurdon Institute, University of Cambridge, Cambridge, CB2 1QN, United Kingdom ${ }^{9}$ Faculty of Science, University of Zurich, Zurich, Switzerland

\section{Abstract}

Computational prediction methods for the identification of microRNA (miRNA) target genes benefit from efficient experimental validation strategies. Here we present a large-scale targeted proteomics approach to validate such predicted miRNA targets in Caenorhabditis elegans. Using selected reaction monitoring (SRM), we quantified 161 proteins of interest in extracts from wildtype and let-7 mutant worms. We demonstrate by independent experimental downstream analyses such as genetic interaction, as well as exemplarily performed polysomal profiling and luciferase assays, that validation by targeted proteomics significantly enriches for biologically relevant let-7 interactors. For example, we show that the zinc finger protein ZTF-7 is a bona fide let-7 miRNA target. We also validated a set of predicted miR-58 targets, demonstrating that this approach is adaptable to multiple miRNAs of interest. We propose that targeted mass spectrometry can be applied generally to validate candidate lists generated by computational methods or by large-scale experiments, and that the described strategy can easily be adapted to other organisms.

\section{Keywords}

targeted proteomics; microRNA targets; let-7; C. elegans; selected / multiple reaction monitoring; $\mathrm{SRM} / \mathrm{MRM}$

\footnotetext{
*Corresponding Authors: Michael O. Hengartner michael.hengartner@imls.uzh.ch Ruedi Aebersold aebersold@imsb.biol.ethz.ch. 10 present address: Biognosys AG, Zurich, Switzerland

11 present address: DKMS Life Science Lab, Dresden, Germany

12 contributed equally

AUTHOR CONTRIBUTIONS M.J., L.R., M.O.H. and R.A. designed the experiments and wrote the paper. L.R. and M.J. did the majority of the data analysis. M.J. did the majority of the experiments. P.P. and V.L. contributed to and supervised the SRM experiments. E.B. contributed to the RNAi and RT-qPCR experiments. B.A.H. and X.C.D. performed the polysomal profiling experiments. C.B. and N.J.L. contributed to the reporter assays. S.P.S. and M.W. provided the C. elegans proteome atlas. H.G. and E.A.M. provided critical input on the manuscript, contributed significantly to the experimental design and the data analysis. M.O.H. and R.A. supervised the whole project.
} 


\section{INTRODUCTION}

miRNAs are short non-coding RNAs that bind to target mRNAs and negatively regulate gene expression. miRNAs play important roles in many developmental and disease-related processes ${ }^{1}$. A full understanding of miRNA function requires knowledge of their target mRNAs. In recent years much progress has been made experimentally and computationally to identify miRNA targets. Indeed, one of the most widely used approaches to identify potential miRNA targets is to apply different target prediction algorithms ${ }^{1}$. However, the many algorithms available predict target sets with only limited overlap and cumulatively identify several hundred potential target mRNAs per miRNA. In addition, large-scale experiments undertaken to identify target mRNAs, such as studies based on mRNA profiling, pulldown of target mRNAs and to a certain extent on genetics also identified a large number of potential miRNA targets ${ }^{2,3}$. Recent publications have clearly shown that using multiple independent experimental approaches greatly improves the reliability of the results obtained ${ }^{4,5}$. Unfortunately, large-scale experiments are often cumbersome and time intensive. Therefore we aimed to establish a targeted quantification method to rapidly validate large numbers of potential miRNA targets.

We reasoned that such a method should measure the most relevant output of gene expression, namely miRNA dependent changes in protein levels of the potential target genes. Moreover, in order to be worthwhile, the method should be easy to use, fast, sensitive, reproducible, quantitative and scalable, as several hundred proteins have to be tested for each miRNA. A technique that promises to fulfill most of those criteria is proteomics. Indeed several groups have shown that shotgun proteomics can be successfully applied to screen for miRNA targets ${ }^{4,6}$. However, with available shotgun proteomics approaches, the bulk of measurement time is spent on signals not arising from the desired candidate proteins. Moreover, many of the desired proteins might not be measured due to the stochastic sampling of the peptide ions that is common to this method. This results in loss of sensitivity and reproducibility to a degree that high confidence data on candidate targets can only be achieved at a high cost of time and labor. In contrast, a targeted proteomics approach such as selected reaction monitoring $(\mathrm{SRM})^{7,8}$ has the potential for fast and reliable protein quantification of candidate genes: By limiting the measurement to the proteins of interest, the sensitivity and the reproducibility of the measurements increase dramatically. This can be achieved by selecting for each candidate miRNA target protein one or several proteotypic peptides (PTPs) - peptides that unambiguously identify a protein of interest and have favorable detection properties by mass spectrometry ${ }^{9}$.

Here, we describe the application of SRM-based targeted proteomics and isotope-coded affinity tag (ICAT) ${ }^{10}$ quantification to screen scores of potential let- 7 targets in C. elegans. Our targeted proteomics approach provided high confidence quantification data, which we then mined to identify potential miRNA targets of biological significance. Independent downstream experiments, including genetic studies, exemplary polysomal profiling and luciferase assays, confirmed that the candidate genes classified as regulated by let- 7 based on our protein quantification data are indeed enriched in let-7interactors. In addition, we showed that the described method is easily adaptable to other miRNAs of interest and quantification strategies than ICAT, such as metabolically labeled worms. Based on this proof of principle study, we suggest that SRM-based targeted proteomics can be widely used to screen candidate lists generated by computational methods and/or by large-scale experiments. 


\section{RESULTS}

\section{SRM-based validation of potential let-7 target genes}

To quantify proteins of interest in a complex whole worm extract generated from $C$. elegans, we developed a protocol combining ICAT sample labeling and SRM mass spectrometry (SRM/ICAT; see Supplementary Fig. 1 and Supplementary Data 1). To test the utility of this new protocol, we applied it to screen through a set of several hundred potential let-7 miRNA targets. We focused on let- 7 because it is highly conserved from $C$. elegans to humans ${ }^{11}$ and is one of the best studied nematode miRNAs ${ }^{12}$. We used for our studies the hypomorphic allele let-7(n2853), which contains a point mutation within the mature let-7 seed sequence that also results in a reduction in let-7 expression levels ${ }^{13}$.

The experimental strategy used to quantify potential let-7targets is described in Figure 1. We developed SRM assays for 181 candidate proteins (from an original list of 861 proteins, including candidates and controls; Fig. 1), and measured the relative abundance changes of these proteins in synchronized let-7(n2853) mutants and wild-type late L4 larvae (when let-7 miRNA expression is the highest) in three biological replicates. Most target proteins (139) could be quantified in all three biological replicates, another 15 in two replicates and 7 in one replicate, yielding quantification data for a majority of the identified proteins (161 out of $181 ; 89 \%$ ) and confirming the high reproducibility of this method (Fig. 2a and

Supplementary Table 2). The normalized $\log 2$ ratios (let-7(n2853) versus wild type) and corresponding $P$-values for all 161 proteins that we quantified are shown in Figure $2 \mathrm{~b}$ and listed in Supplementary Table 2.

Twenty nine proteins showed a significant difference in expression level $(P<0.01$, one sample Student's t-test; Fig. 2c and Supplementary Table 3): 10 proteins were down- and 19 proteins upregulated in let-7(n2853) worms. As expected, the two control genes vit-2 and vit-6, which show a greatly reduced abundance at the mRNA level in let-7(n2853) mutants (Hurschler B et al., unpublished data) were also strongly downregulated in our assay (13fold and 23-fold respectively; Supplementary Table 3). The upregulated protein set included LET-526 (also known as LSS-4), the only previously reported let- 7 target $^{14}$ whose abundance we could measure. Interestingly, our SRM measurements suggest that the two splice-variants of LET-526 respond differently to let-7: whereas a peptide specific for the LET-526a splice form showed a strong, 3.1-fold change, a second peptide, matching to a region common to both splice-isoforms, displayed only a weak 1.2-fold upregulation in let-7(n2853) mutant worms (see Supplementary Fig. 2a and b). We confirmed and validated this splice-variant specific response by polysomal profiling (see Supplementary Fig. 2c and Supplementary Data 2).

Also among the significantly changed proteins were 15 of the 66 predicted let- 7 targets based on bioinformatics, and 9 out of 53 proteins whose mRNA do not contain a predicted let-7 target site, but that have been linked to let-7 through other experimental approaches or the literature (Supplementary Table 3). By contrast, only 2 out of 39 of the randomly picked "neutral controls" showed a significant abundance change. Interestingly the "neutral controls" were the only significantly underrepresented group among the regulated proteins $(P=0.016$, Fisher's Exact Test). This low "hit rate" for these randomly tested proteins confirms that our initial candidate list was indeed enriched for let-7 miRNA target genes.

Whether the regulated candidates are primary or secondary targets of let-7 cannot be determined from the protein ratios. Although the most straightforward explanation for proteins downregulated in let-7(n2853) mutants is secondary effects, as it is the case for the two control proteins VIT-2 and VIT-6 (Hurschler B et al., unpublished data), miRNAs have recently been reported to act as positive regulators under certain conditions ${ }^{15}$. A gain-of- 
function caused by the point mutation within the seed region of the mature let-7 miRNA in let-7(n2853) mutants, resulting in better binding to a suboptimal seed sequence, also cannot be excluded at this point.

\section{SRM-based validation enriches for let-7 genetic interactors}

let-7(n2853) mutant worms grown at $25^{\circ} \mathrm{C}$ die at the adult stage due to vulval bursting. Knockdown by RNAi of known let-7 miRNA targets has been shown in some cases to rescue this lethality to different extents ${ }^{14}$. To determine whether the positive hits in our proteomics screen are indeed enriched in let-7 targets, we knocked-down all 29 genes that showed protein changes in let-7(n2853) mutants (up- or downregulated) to determine whether they could suppress the let-7(n2853) lethal phenotype. Knock-down of ten genes successfully and reproducibly rescued the lethality to at least $20 \%$ (Fig. 3a and Supplementary Table 4). As expected, the vast majority (9/10) of the suppressors were genes that were upregulated in let-7(n2853) mutants (and whose overexpression could thus be compensated via RNAi knock-down). As a control, we performed a similar experiment using 29 candidate genes that did not show significant protein changes in our targeted proteomics assay. Only three genes from this set were able to rescue the lethality (Fig. 3b and Supplementary Table 5), demonstrating that the regulated protein set is significantly enriched for genes that genetically interact with let-7 $(P=0.024$ for all regulated genes, $P=$ 0.013 considering only the upregulated genes, Fischer's Exact Test; Supplementary Fig. 3). We conclude that a targeted proteomics method can indeed be used to enrich for miRNA interaction partners from a list of candidate genes.

\section{Partial correlation between protein and mRNA abundances}

In addition to causing translational repression, miRNAs can also lead to degradation of their targets ${ }^{1}$. To determine whether the changes in protein levels that we observed could also be captured at the mRNA level, we determined transcript levels for all 161 proteins, as well as for the well established let-7 targets daf-12 and lin-41, in wild-type and let-7(n2853) worms (Supplementary Table 6). Both daf-12 and lin-41 mRNA levels were significantly upregulated in let-7(n2853) mutants ( $P=0.006$ and 0.048 , respectively, one-sided one sample Student's t-test), as has been reported previously ${ }^{16,17}$. Interestingly, while some proteins showed a very good correlation between mRNA and protein changes, others, including the known let-7 target let-526, showed a significant protein change but no strong change in mRNA levels (Fig. 4a). As our targeted proteomics method cannot distinguish primary from secondary targets, we do no know at this point whether the proteins that show strong protein changes but no mRNA changes are primary let-7 targets.

We next focused our attention on the 47 genes that we previously tested for suppression of let-7(n2853) lethality (see above). Whereas many of the 13 RNAi suppressors showed large changes in protein levels in let-7(n2853) mutants, their mRNA levels varied only weakly if at all (Fig. 4b). We conclude that many of the protein changes we detected in our targeted proteomics approach are not recapitulated on the mRNA level, and that while mRNA profiling can identify a large number of primary targets ${ }^{4,5}$, it would fail to detect several of the biologically important candidates revealed by protein quantification.

\section{The zinc finger protein ZTF-7 is a bona fide let-7 target}

One of the most interesting candidates from our RNAi screen was $z t f-7$ (F46B6.7), as knockdown of this gene not only suppressed lethality (see Fig. 3 and Supplementary Table 4), but also the sterility observed in let-7(n2853) mutants at $25^{\circ} \mathrm{C}$ (data not shown). Of the genes that we tested, only the two positive controls $d a f-12$ and lin- 41 could also suppress both defects. Consistent with our RNAi results, lethality was strongly reduced in 
ztf-7(tm600); let-7(n2853) double mutant worms when compared to the let-7(n2853) single mutants (Fig. 5a).

Our targeted proteomics measurements had indicated that ZTF-7 protein levels are elevated by $\sim 10 \%$ in let-7(n2853) mutants when compared to wild-type worms. Although this increase is admittedly mild, it was reproducible across all three biological replicates and highly significant ( $P=0.005$, one sample Student's t-test; see Supplementary Table 3).

As $z t f-7$ is predicted to contain a conserved perfect seed complementary let-7binding site in its $3^{\prime} \mathrm{UTR}^{18,19}$ we next tested whether the $z t f-73^{\prime} \mathrm{UTR}$ is able to confer let- 7 dependent regulation of a reporter transcript. It has been reported that certain $C$. elegans $3^{\prime}$ UTRs can elicit a miRNA dependent response in human cell lines ${ }^{20}$. As the sequence of the mature let-7 miRNA is identical in $C$. elegans and in humans ${ }^{11}$, we could rapidly test the effect of both overexpression and depletion of human let-7a miRNA in HeLa cells, which were transfected with a dual luciferase plasmid where the $z t f-73^{\prime}$ UTR was cloned directly downstream of the firefly luciferase gene (luciferase::ztf-7 $3^{\prime}$ UTR). Indeed, we observed a strong response of the luciferase::ztf-7 $3^{\prime}$ UTR reporter to both human let-7a up- and downregulation (Fig. 5b).

Taken together, our proteomics, genetic and reporter assays strongly suggest that $z t f-7$ is a bona fide let-7 miRNA target. Moreover, $z t f-7$ was also identified recently as a potential let-7 target by novel approach ${ }^{21}$, thus providing independent support for our claim that $z t f-7$ is a bona fide let-7 target. Additionally that method also allowed to map the binding site, which overlapped perfectly with the predicted conserved seed site. Further work will be required to understand the function of ZTF-7 in C. elegans development.

\section{A streamlined pipeline for miRNA target validation}

To test the generality of our targeted proteomics approach, we performed a second experiment in which we aimed to validate predicted targets of miR-58. mir-58 is of particular interest as it is part of a miRNA gene family. Whereas the single mutants show no obvious defect, the whole family knock out is severely sick ${ }^{22}$. Therefore, it was unclear whether predicted miR-58 targets would already show significant changes in single mutants.

We took advantage of this second experiment to introduce several technical improvements. First, to target the full peptide repertoire of $C$. elegans and not just cysteine-containing peptides, we used metabolically heavy isotope-labeled worms ${ }^{23}$ as a quantification standard. Second, we used crude chemically synthesized peptides to establish and optimize the SRM assays ${ }^{24}$. Third, we applied a newly developed algorithm that automatically assigns peak groups to their corresponding peptides and controls the false discovery rate (FDR) of those assignments (Reiter L., Rinner O. et al., manuscript submitted).

The TargetScan ${ }^{18,19}$ program predicts 118 miR-58 target genes in $C$. elegans. To validate this candidate list, we ordered crude synthetic peptides for all 118 proteins and used them to develop and optimize SRM assays. We also developed SRM assays for a set of 42 "neutral control" genes that were randomly selected from a set of 5000 genes that are well expressed in L4 hermaphrodites (data not shown). We next measured as many of these proteins as possible directly in complex, unfractionated extracts derived from staged L4 wild-type and mir-58(n4640) mutant worms. Applying a 5\% FDR cutoff for the correct peak assignment, we could quantify 27 out of the 118 predicted targets and 24 out of the 42 randomly chosen proteins in at least one replicate pair (Supplementary Table 7). We found that predicted miR-58 targets were, as a group, significantly more likely to be upregulated in mir-58(n4640) mutant worms when compared to the randomly chosen control group (Fig. 6; $P<10^{-3}$, Kolmogorov-Smirnov (KS) test). Consistent with this observation, we found that 4 
out of 27 predicted targets, compared to 1 out of 24 control proteins, were significantly upregulated in mir-58(n4640) mutant worms $(P<0.05$, one-sided one sample Student's ttest; Supplementary Table 7)

miR-58 is a member of a highly abundant miRNA family that also includes miR-80, miR-81, miR-82, and the recently discovered miR-183422. As expected, TargetScan predicts largely overlapping sets of targets for the various miR-58 family members. Thus, the miR-58 family might show significant redundancy, as loss of a single miRNA might be compensated by the other family members. Consistent with this hypothesis, single mir-58 family mutants are all overtly wild-type, whereas the mir-58,80,81,82 quadruple mutant is severely sick. Interestingly, despite this redundancy at the organismal level, we found that targets predicted to be bound by all miR-58 family members (18 out of 27) still showed a significant increase in abundance in mir-58(n4640) mutants (Fig. 6; $P<10^{-3}$, KS test). Analysis of protein levels in the family knock-out will be necessary to determine the exact extent of compensation that does occur, if any, within this miRNA family.

\section{DISCUSSION}

Our results demonstrate the suitability and advantages of a targeted proteomics approach to find biologically relevant candidate miRNA targets. First, this method measures changes in protein levels, arguably the most relevant assay for miRNA activity. Second, our approach allows for the quantification of several hundred proteins and thus has a much higher throughput than traditional protein quantification methods such as immunoblotting. Additionally the development of suitable mass spectrometric assays is significantly faster and cheaper than that of immunoassays ${ }^{25}$. Moreover, once an SRM assay is established for a protein, it becomes universally useful and exportable ${ }^{8,26}$. Thus, the SRM assays we established for the 312 C. elegans proteins that we measured (Supplementary Table 8 and 9) can now readily be used by others. Third, because it focuses on highly responsive peptides, our SRM-based approach is highly sensitive and reproducible. Indeed, we could reproducibly measure changes as small as ten percent in total protein abundance, as exemplified with ZTF-7. This high accuracy is particularly important in the analysis of potential miRNA targets, as miRNAs have been shown to mostly induce small changes in target gene expression ${ }^{4,6}$.

Despite the clear value of our targeted proteomics approach, several challenges remain. First, although we achieved a high sensitivity, we still failed to quantify a substantial fraction of the proteins we had in our target list. Further technical improvements, e.g., combination of chemically synthesized peptides and sample fractionation - could potentially boost the sensitivity by an order of magnitude, as has previously been shown in yeast ${ }^{7}$. Second, the targeted proteomics approach cannot distinguish primary from secondary targets. Additional experiments will thus invariably be necessary to establish which hits are direct targets, as we did for $z t f-7$.

We must also stress that the applicability of our targeted proteomics method to whole organ or whole animals is particularly challenging, as the miRNA of interest might be of low abundance or have a highly restricted expression pattern. We therefore conclude that our method will function best in situations where sufficient material can readily be obtained and the sample is homogenous (e.g., cell lines). For a more in depth discussion of those three limitations please refer to the Supplementary Discussion.

The targeted proteomics approaches described here should be considered complementary to the shotgun proteomics approaches recently reported to identify miRNA targets ${ }^{4,6}$. While shotgun proteomics should be regarded as one of several discovery tools that can be used to 
find potential new miRNA target candidates, a targeted proteomics approach should be perceived as a validation / hypothesis driven tool with high sensitivity, reproducibility and accuracy.

Importantly, while we applied the targeted proteomics method described here for the validation of miRNA targets in $C$. elegans, the method is broadly applicable, and can readily be adapted to other organisms and to other biological questions. A wide range of quantification methods are available ${ }^{27}$, suitable for nearly every extract composition. In addition, there are public proteomics databases for a wide range of different organisms, where experimentally identified proteins and their corresponding PTPs can be easily mined $^{28,29}$. Even for organisms where such proteomics data is not readily accessible, sophisticated PTP prediction algorithms ${ }^{9}$ can be consulted in order to target the right peptides. Thus, the targeted proteomics approach described here can be applied generally to measure protein abundances of long candidate lists generated by computational methods or by large-scale experiments.

\section{MATERIALS and METHODS}

\section{Mutations}

All mutants used in this study were derived from the wild-type variety Bristol strain N2. The following mutations were used: LGV: ztf-7(tm600) (see http://www.wormbase.org); LGX: let-7(n2853)13, ain-1(ku322) 32 .

\section{Sample preparation for let-7 related experiments}

C. elegans strains were cultured as described previously ${ }^{33}$. All strains were grown at either $15^{\circ} \mathrm{C}$ or $25^{\circ} \mathrm{C}$.

C. elegans wild-type strain N2 (Bristol) and the let-7(n2853) mutant strain MT7626 were grown on 9-cm nematode growth medium (NGM) agar plates seeded with a lawn of the $E$. coli strain OP50. N2 and let-7(n2853) worms were always grown in parallel (3 biological replicates total). Protein extracts were generated from synchronized late L4 larval stage worms (before vulval bursting), which were grown at $25^{\circ} \mathrm{C}$. Worms were harvested and washed three times in M9 media. Generation of the protein extract has been described previously ${ }^{30}$. The buffer used was $50 \mathrm{mM}$ Tris/ $\mathrm{HCl}$ (pH 8.3), $5 \mathrm{mM}$ EDTA, $8 \mathrm{M}$ urea and $0.125 \%$ SDS. Cell debris were removed by sequential centrifugation ( $4000 \mathrm{~g}$ for $5 \mathrm{~min}$ followed by $16000 \mathrm{~g}$ for $5 \mathrm{~min}$ ) and the protein concentrations of the purified extracts were determined by using the Bradford reagent (Sigma-Aldrich). The protein concentrations of $\mathrm{N} 2$ and let-7(n2853) extracts were adjusted to each other in order to minimize biases for the subsequent ICAT (Applied Biosystems) labeling ${ }^{10}$.

ICAT labeling, tryptic digestion of the samples, and the isolation and clean up of ICAT labeled cysteine containing peptides were performed as described in Shiio and Aebersold $(2006)^{34}$. N2 extracts were always labeled with the heavy ICAT reagent and let-7(n2853) extracts with the light ICAT reagent. A total of $5 \mathrm{mg}$ per sample and replicate was labeled, resulting in approximately $500 \mu \mathrm{g}$ ICAT-labeled peptides. The peptide mixtures were cleaned by Sep-Pak tC18 cartridges (Waters) and eluted with $60 \%$ acetonitrile. The resulting peptide samples were separated according to the isoelectric point of the peptides by off-gel electrophoresis (OGE) 7 using a pH 3-10 IPG strip (AmershamBiosciences), and a 3100 OFFGEL Fractionator (AgilentTechnologies) with collection in 24 wells. Prior to electrofocusing, the peptides were evaporated to dryness in a centrifugal vacuum concentrator and solubilized in a separation medium containing $7 \mathrm{M}$ Urea, $2 \mathrm{M}$ thiourea, 1 $\%$ w/v DTT, $5 \%$ v/v glycerol, $1 \%$ v/v carrier ampholytes mixture (IPG buffer $\mathrm{pH} 3.0-10.0$, GE Healthcare) loaded in all wells and the potential was fixed at $8000 \mathrm{~V}$ with a maximum 
current set at $50 \mu \mathrm{A}$. Peptides collected in each well were cleaned by Sep-Pak tC18 cartridges (Waters) and eluted with $60 \%$ acetonitrile. All peptide samples were evaporated in a vacuum centrifuge to dryness, resolubilized in $2 \%$ acetonitrile and $0.1 \%$ formic acid and frozen at $-20{ }^{\circ} \mathrm{C}$ until analysed on the mass spectrometer.

\section{RT-qPCR}

Prior to protein isolation, a small aliquot of intact worms of each biological replicate (three times N2 wild-type worms and three times let-7(n2853) worms - see "sample preparation" above) was frozen, and subsequently used for total RNA isolation. Total RNA was isolated using the Nucleo Spin RNA II kit (Marcherey-Nagel) according to the manufacturer's instructions. After total RNA isolation, genomic DNA was further digested by DNase I using the Turbo DNA-free kit (Ambion) according to the manufacturer's instructions. Total RNA concentrations were determined with the Nanodrop device (Thermo Fisher Scientific). RNA reverse transcription (RT) was performed using the Transcriptor High Fidelity cDNA Synthesis Kit (Roche) with oligo-(dT) primers, according to the manufacturer's recommendations using equal amounts of RNA $(4 \times 2 \mu \mathrm{g})$ for each sample. qPCR reactions were performed in technical duplicate for each of the biological triplicates using MESA Green qPCR Mastermix Plus for SYBR Assay (Eurogentec), according to the manufacturer's recommendations, on an ABI 7900 HT Sequence Detection System coupled to ABI Prism 7900 SDS 2.2 Software (Applied Biosystems). Relative transcript levels were calculated using the $2^{-\Delta C t}$ method $^{35}$. Most primer pairs were designed via the Roche Universal Probe Library. All the primer pairs used are listed in Supplementary Table 10.

\section{Polysomal profile analysis and subsequent RT-qPCR}

The polysomal profile analysis and subsequent RT-qPCR was performed using the same polysomal fractions and protocols as Ding XC and Grosshans H (2009) ${ }^{17}$. The experiments were performed in triplicate.

We could not develop an RT-qPCR assay specific for let-526b only, as there is just a small region $(<50 \mathrm{bp})$ in this splice form that is not present in let-526a. Instead we used primers specific for both splice forms. The primers used for let-526a specifically were $5^{\prime}$ accacgaccaccatatccat $3^{\prime}$ and $5^{\prime}$ cgggcattgtagaagagagc $3^{\prime}$. The primers for both let-526a and let-526b were $5^{\prime}$ tcgecgagagattactcgtt $3^{\prime}$ and $5^{\prime}$ agaagcgatgcaaagagcat $3^{\prime}$

\section{RNAi experiments}

Gene knockdown was achieved through RNAi by feeding as published $36,37,38,39,14$. Media supplement were used at the following concentrations: ampicillin, $200 \mu \mathrm{g} / \mathrm{ml}$; isopropyl- $\beta$ D-thiogalactopyranoside (IPTG), $2 \mathrm{mM}$. All the experiments were performed at $25^{\circ} \mathrm{C}$. About 100-150 synchronized L1s were placed on IPTG/AMP NGM agarose plates seeded with $200 \mu \mathrm{l} E$. coli expressing double-stranded RNA (dsRNA). The worms were scored 72 hours later (adult stage) for suppression of lethality. Clones were regarded as positive when at least $20 \%$ of the worms were viable as adults. All the clones used were verified by sequencing for their correct insert. All RNAi plasmids used are listed in Supplementary Table 11.

\section{Lethality assays for $C$. elegans mutant strains}

All the experiments were performed at $25^{\circ} \mathrm{C}$ and in quadruplicate. About $100-150$ synchronized L1s were placed on NGM agarose plates seeded with $250 \mu 1$ OP50 E. coli bacteria. The worms were scored 48 hours later (= 12 hours post L4) for suppression of lethality. Following strains were tested: MT7626 (let-7(n2853)), FX00600 (ztf-7(tm600)), 
WS5673 (ztf-7(tm600); let-7(n2853)). At least $20 \%$ of the worms had to be viable in the double mutant worms (WS5673) in order to be regarded as a successful suppressor.

It should be noted that 24 hours post L4 most double mutant worms (WS5673) were dead, therefore suggesting more a lethality delay than a true suppression. A developmental delay in WS5673 worms could be excluded as the survivors at the 12 hours post L4 time point had fully developed gonads with oocytes and at least $60 \%$ of the survivors also had embryos.

\section{Cloning of 3' UTRs from candidate genes}

pEM393 is a dual luciferase Gateway (Invitrogen) compatible vector, adapted from the psiCHECK-II vector (Promega). The 3'UTRs of F46B6.7 (ztf-7), C12C8.3 (lin-41), F11A1.3a (daf-12), F13D11.2 (hbl-1), F36A4.7 (ama-l) and T04C12.6 (act-l) were cloned directly downstream of the Firefly Luciferase gene. The $3^{\prime}$ UTRome $C$. elegans database ${ }^{40}$ (utrome.org) and Wormbase (www.wormbase.org) were used to retrieve the sequences for the $3^{\prime}$ UTRs of interest. Supplementary Table 12 lists the primers used for the PCR reaction and the length of each putative $3^{\prime}$ UTR sequence cloned. Gateway cloning was performed according to the manufacturer's instructions (Invitrogen). Briefly, the sequences of interest were amplified using the $a t t B$ adapter primer PCR protocol to generate PCR clones containing the $3^{\prime}$ UTR flanked by respective attB sites (attB1 site at the $5^{\prime}$ end and the attB2 site the $3^{\prime}$ end). The PCR product was recombined into pDONR221 by the BP reaction to create the entry clone set (see Supplementary Table 12). The entry clones were verified by sequencing and then recombined with the destination vector pEM393 to generate the expression clone set via the LR reaction (see Supplementary Table 12). The expression clones were again verified by sequencing and used for the subsequent luciferase assays.

\section{Luciferase assay}

The reactions were performed in 96 well plates. miRNA mimics or inhibitors were ordered from Dharmacon. $150 \mathrm{ng}$ of the dual luciferase expression clone containing the $3^{\prime}$ UTR of interest and $10 \mathrm{pmol}$ of the either the human let-7a mimic, the control mimic (C. elegans miR-67), the human let-7a inhibitor or the control inhibitor (against $C$. elegans miR-67) were transfected into HeLa cells (10,000 cells per reaction) in triplicate. The Dual-Glo Luciferase assay system (Promega) was used two days post-transfection, according to the manufacturer's instructions. All the firefly luciferase readouts were first normalized to their matching renilla luciferase readouts. Those readouts were further normalized to empty vector (pEM393 vector without any $3^{\prime}$ UTR) controls and then the let-7a readouts (mimics and inhibitors) were normalized to their respective oligo controls.

\section{Selection of let-7 candidates}

861 genes of interest were selected based on literature, computational prediction algorithms ${ }^{41,42,43,14,44}$, experimental evidence, published literature, including known let-7 target genes ${ }^{13,14,45,46,47,48,49,50,51}$ and MS detectability ${ }^{30}$ (random controls). The predicted targets from the computational prediction algorithms were (1) from miRBase - based on the miRanda prediction algorithm v3.0 ${ }^{\text {Ref.43 }}$. The let- 7 targets were downloaded on 25/April/ 2006. A $P$-value cutoff of 0.005 was applied; (2) from Pictar - all let-7 targets available on the website (http://pictar.mdc-berlin.de/cgi-bin/new_PicTar_nematode.cgi? species=nematode) when download (26/April/2006) ${ }^{41}$; (3) our so called "Stark targets" let-7 target prediction for $C$. elegans based on the algorithm described in Stark et al. $(2005)^{42}$. The target list was provided by Alexander Stark; (4) all the let-7 targets published in Grosshans et al. $(2005)^{14}$; (5) all the let-7 targets published in Watanabe et al. $(2006)^{44}$. 


\section{Design of SRM assays for let-7 related experiments}

PTPs were selected based on a large shotgun proteomics data set ${ }^{30}$. This $C$. elegans proteome atlas data set was filtered for a peptide-spectrum match false discovery rate of 0.17 $\%$ corresponding to a protein identification false discovery rate of $5 \%{ }^{31}$. Proteotypic peptides needed to contain at least one cysteine ${ }^{10}$ and doubly charged peptides with a high number of identifications were preferred. For each peptide, 4 to 8 fragment ions from the yion series were computed. Fragment ions (Q3) with an $\mathrm{m} / \mathrm{z}$ above the peptide ion (Q1) and with a defined minimal distance to the peptide ion were chosen $\left(\mathrm{m} / \mathrm{z}_{\mathrm{Q} 3}-\mathrm{m} / \mathrm{z}_{\mathrm{Q} 1} \geq 50\right.$ Thomson ). The peptide ion/fragment ion (Q1/ Q3) transitions were used to trigger the acquisition of triple quadrupole (QQQ) MS/MS spectra of the peptides of interest in $C$. elegans whole worm extracts and in off gel electrophoresis (OGE) fractionated samples. Proteotypic peptides for additional 19 proteins not contained in the $C$. elegans proteome atlas were found using SRM triggered MS/MS. For the samples derived from the OGE fractionations, the isoelectric points of the peptides were predicted using BioPerl ${ }^{52}$ and peptides were targeted in the predicted fraction and in the two neighboring fractions if available.

\section{Database search and extraction of optimal SRM transitions for let-7 related experiments}

The data was converted from the raw .wiff to the .mzXML format using the program mzWiff (version 3.5.3, build Apr 162008 14:40:24). The MS/MS spectra from the SRM triggered MS/MS experiments were searched against wormpep140 (www.wormbase.org) using Sequest on a Sorcerer machine (SorcererTM-SEQUEST®, 3.10.4 release) with light ICAT as static modification and heavy ICAT and/or. oxidized methionine as variable modifications. Precursor mass tolerance was set to $1.5 \mathrm{Da}$ and the data were searched fully tryptic with maximal two missed cleavages. The data was filtered with a peptide-spectrum match FDR of $2.5 \%$ using PeptideProphet ${ }^{53}$. Three transitions for each proteotypic peptide were generated by extracting the three highest fragment ions and the retention time of the peptide from the triple quadrupole MS/MS. All transitions used for quantification in this study are listed in Supplementary Table 8.

\section{Mass spectrometry analysis of let-7 related experiments}

All peptide samples were analyzed on a hybrid triple quadrupole/ion trap mass spectrometer (4000QTrap, ABI/MDS-Sciex) equipped with a nanoelectrospray ion source.

Chromatographic separations of peptides were performed on a Tempo nano LC system (Applied Biosystems) coupled to a $16 \mathrm{~cm}$ fused silica emitter, $75 \mu \mathrm{m}$ diameter, packed with a Magic C18 AQ $5 \mu \mathrm{m}$ resin (Michrom BioResources). Peptides were loaded onto a trapping column from a cooled $\left(4{ }^{\circ} \mathrm{C}\right)$ Tempo autosampler and separated with a linear gradient of acetonitrile/water, containing $0.1 \%$ formic acid, at a flow rate of $300 \mathrm{nl} / \mathrm{min}$. A gradient from 5 to $30 \%$ acetonitrile in 30 or 60 minutes was used. Collision energies used for both SRM and MS/MS analyses were calculated according to the formulas: $\mathrm{CE}=0.044 *$ $\mathrm{m} / \mathrm{z}+5.5$ or $\mathrm{CE}=0.051 * \mathrm{~m} / \mathrm{z}+0.5(\mathrm{CE}$, collision energy, $\mathrm{m} / \mathrm{z}$, mass-to-charge ratio of the precursor ion) for doubly and triply charged precursor ions, respectively ${ }^{7}$.

In the SRM assays validation phase, the mass spectrometer was operated in SRM mode, triggering acquisition of a full MS/MS spectrum upon detection of an SRM trace (MRMtriggered MS/MS, threshold 200 ion counts). The set of SRM transitions generated as previously described was split into multiple MS-methods and analyzed in several runs. Each SRM acquisition was performed with Q1 and Q3 operated at unit resolution $(0.7 \mathrm{~m} / \mathrm{z}$ half maximum peak width). An average of 60 transitions (dwell time $20 \mathrm{~ms} /$ transition) per run was used for the measurements. MS/MS spectra were acquired in enhanced product ion (EPI) mode for the two highest SRM transitions, using dynamic fill time, Q1 resolution low, scan speed $4000 \mathrm{amu} / \mathrm{s}, \mathrm{m} / \mathrm{z}$ range 300-1400. 
The complete transition list used for the quantifications is shown in Supplementary Table 8. An average of 60 transitions per run was used for the measurements. The quantification measurements were done in the scheduled SRM mode (retention time window: 900 seconds; target scan time: 2 seconds)

\title{
Quantitative and statistical analysis of the let-7 related experiments
}

Peak height for the transitions associated to the let-7(n2853) (light ICAT label) and wildtype (heavy ICAT label) derived peptides were quantified using the software MultiQuant v. 1.1 Beta (Applied Biosystems). Log2 fold changes were calculated for each transition separately. These values were then normalized using 11 proteotypic peptides (see Supplementary Fig. 1a and Supplementary Fig. 4) on each biological replicate separately. To test for statistically significant abundance changes, a two sided one sample t-test was done on the normalized $\log 2$ fold changes of the transitions grouped according to protein $(\mu$ equal to zero). To generate our list of regulated candidates we used a $P \leq 0.01$ cutoff.

\section{miR-58 related experiments}

Details are given in "Supplementary Materials and Methods"

\section{Supplementary Material}

Refer to Web version on PubMed Central for supplementary material.

\section{Acknowledgments}

\begin{abstract}
We thank R. Ketting (Hubrecht Institute, Utrecht) and B. Tops (Utrecht University, Utrecht) for providing the metabolically labeled $C$. elegans sample. We also thank A. Stark (Research Institute of Molecular Pathology, Vienna) for sharing miRNA target predictions for $C$. elegans. Additionally we thank M. Moser for the assistance with the RT-qPCR assays; B. Roschitzky and B. Gerrits for technical support; H. Rehrauer for statistical support; R. Schlapbach for access to the Functional Genomics Center Zurich; the Hengartner, Aebersold, Grosshans and Miska laboratories, Erich Brunner and the whole Q-MOP team for insightful discussion and comments on the manuscript. This work was funded in part by the University of Zurich Research Priority Program in Systems Biology/Functional Genomics, the Swiss National Science Foundation, the GEBERT RÜF Foundation, SystemsX, the Ernst Hadorn Foundation and the ETH Zurich. M.J. and L.R. were supported by a grant from the Research Foundation of the University of Zurich. M.J. was also supported by a fellowship from the Roche Research Foundation. P.P. is the recipient of a Marie Curie Intra-European fellowship. V.L. was supported by a grant from F. Hoffmann-La Roche Ltd. to the Competence Center for Systems Physiology and Metabolic Diseases. H.G. was supported by the Swiss National Research Foundation, the Novartis Research Foundation and by an ERC Starting Investigator Grant (miRTurn). X.C.D was supported by a Boehringer Ingelheim Funds PhD Student fellowship. C.B., N.J.L and E.A.M. were supported by a Cancer Research UK Programme Grant to E.A.M. We also want to apologize to all the authors in the miRNA and proteomics field who we did not cite due to limited space.
\end{abstract}

\section{REFERENCES}

1. Bartel DP. MicroRNAs: target recognition and regulatory functions. Cell. 2009; 136:215-33. [PubMed: 19167326]

2. Lai EC. miRNAs: whys and wherefores of miRNA-mediated regulation. Curr. Biol. 2005; 15:R458460. [PubMed: 15964265]

3. Ørom UA, Lund AH. Experimental identification of microRNA targets. Gene. 2010; 451:1-5. [PubMed: 19944134]

4. Baek D, et al. The impact of microRNAs on protein output. Nature. 2008; 455:64-71. [PubMed: 18668037]

5. Hendrickson DG, et al. Concordant regulation of translation and mRNA abundance for hundreds of targets of a human microRNA. PLoS Biol. 2009; 7:e1000238. [PubMed: 19901979]

6. Selbach M, et al. Widespread changes in protein synthesis induced by microRNAs. Nature. 2008; 455:58-63. [PubMed: 18668040] 
7. Picotti P, Bodenmiller B, Mueller LN, Domon B, Aebersold R. Full Dynamic Range Proteome Analysis of S. cerevisiae by Targeted Proteomics. Cell. 2009 doi:10.1016/j.cell.2009.05.051.

8. Picotti P, et al. A database of mass spectrometric assays for the yeast proteome. Nat Methods. 2008; 5:913-4. [PubMed: 18974732]

9. Mallick P, et al. Computational prediction of proteotypic peptides for quantitative proteomics. Nat Biotechnol. 2007; 25:125-131. [PubMed: 17195840]

10. Gygi SP, et al. Quantitative analysis of complex protein mixtures using isotope-coded affinity tags. Nat Biotechnol. 1999; 17:994-9. [PubMed: 10504701]

11. Pasquinelli AE, et al. Conservation of the sequence and temporal expression of let-7 heterochronic regulatory RNA. Nature. 2000; 408:86-9. [PubMed: 11081512]

12. Bussing I, Slack F, Groshans H. let-7 microRNAs in development, stem cells and cancer. Trends in Molecular Medicine. 2008; 14:400-409. [PubMed: 18674967]

13. Reinhart BJ, et al. The 21-nucleotide let-7 RNA regulates developmental timing in Caenorhabditis elegans. Nature. 2000; 403:901-6. [PubMed: 10706289]

14. Groshans H, Johnson T, Reinert K, Gerstein M, Slack F. The Temporal Patterning MicroRNA Regulates Several Transcription Factors at the Larval to Adult Transition in. Developmental Cell. 2005; 8:321-330. [PubMed: 15737928]

15. Vasudevan S, Tong Y, Steitz JA. Switching from repression to activation: microRNAs can upregulate translation. Science. 2007; 318:1931-4. [PubMed: 18048652]

16. Bagga $\mathrm{S}$, et al. Regulation by let-7 and lin-4 miRNAs results in target mRNA degradation. Cell. 2005; 122:553-63. [PubMed: 16122423]

17. Ding XC, Grosshans H. Repression of C. elegans microRNA targets at the initiation level of translation requires GW182 proteins. EMBO J. 2009; 28:213-22. [PubMed: 19131968]

18. Lewis BP, Burge CB, Bartel DP. Conserved seed pairing, often flanked by adenosines, indicates that thousands of human genes are microRNA targets. Cell. 2005; 120:15-20. [PubMed: 15652477]

19. Ruby J, et al. Large-Scale Sequencing Reveals 21U-RNAs and Additional MicroRNAs and Endogenous siRNAs in C. elegans. Cell. 2006; 127:1193-1207. [PubMed: 17174894]

20. Nottrott S, Simard MJ, Richter JD. Human let-7a miRNA blocks protein production on actively translating polyribosomes. Nat Struct Mol Biol. 2006; 13:1108-1114. [PubMed: 17128272]

21. Andachi Y. A novel biochemical method to identify target genes of individual microRNAs: Identification of a new Caenorhabditis elegans let-7 target. RNA. 2008; 14:2440-2451. [PubMed: 18824511]

22. Alvarez-Saavedra E, Horvitz HR. Many Families of C. elegans MicroRNAs Are Not Essential for Development or Viability. Curr Biol. 2010 doi:10.1016/j.cub.2009.12.051.

23. Krijgsveld J, et al. Metabolic labeling of C. elegans and D. melanogaster for quantitative proteomics. Nat Biotechnol. 2003; 21:927-931. [PubMed: 12858183]

24. Picotti P, et al. High-throughput generation of selected reaction-monitoring assays for proteins and proteomes. Nat. Methods. 2010; 7:43-46. [PubMed: 19966807]

25. Picotti P, et al. High-throughput generation of selected reaction-monitoring assays for proteins and proteomes. Nat. Methods. 2009 doi:10.1038/nmeth.1408.

26. Addona TA, et al. Multi-site assessment of the precision and reproducibility of multiple reaction monitoring-based measurements of proteins in plasma. Nat. Biotechnol. 2009 doi:10.1038/nbt. 1546.

27. Mueller LN, Brusniak M, Mani DR, Aebersold R. An assessment of software solutions for the analysis of mass spectrometry based quantitative proteomics data. J Proteome Res. 2008; 7:51-61. [PubMed: 18173218]

28. Brunner E, et al. A high-quality catalog of the Drosophila melanogaster proteome. Nat Biotechnol. 2007; 25:576-583. [PubMed: 17450130]

29. Baerenfaller K, et al. Genome-scale proteomics reveals Arabidopsis thaliana gene models and proteome dynamics. Science. 2008; 320:938-41. [PubMed: 18436743]

30. Schrimpf SP, et al. Comparative Functional Analysis of the Caenorhabditis elegans and Drosophila melanogaster Proteomes. PLoS Biol. 2009; 7:e48. [PubMed: 19260763] 
31. Reiter L, et al. Protein identification false discovery rates for very large proteomics datasets generated by tandem mass spectrometry. Mol. Cell Proteomics. 2009 doi:10.1074/mcp.M900317MCP200.

32. Ding L, Spencer A, Morita K, Han M. The Developmental Timing Regulator AIN-1 Interacts with miRISCs and May Target the Argonaute Protein ALG-1 to Cytoplasmic P Bodies in. Molecular Cell. 2005; 19:437-447. [PubMed: 16109369]

33. Brenner S. The genetics of Caenorhabditis elegans. Genetics. 1974; 77:71-94. [PubMed: 4366476]

34. Shiio Y, Aebersold R. Quantitative proteome analysis using isotope-coded affinity tags and mass spectrometry. Nat Protoc. 2006; 1:139-145. [PubMed: 17406225]

35. Livak KJ, Schmittgen TD. Analysis of relative gene expression data using real-time quantitative PCR and the 2(-Delta Delta C(T)) Method. Methods. 2001; 25:402-8. [PubMed: 11846609]

36. Fraser AG, et al. Functional genomic analysis of C. elegans chromosome I by systematic RNA interference. Nature. 2000; 408:325-30. [PubMed: 11099033]

37. Kamath RS, et al. Systematic functional analysis of the Caenorhabditis elegans genome using RNAi. Nature. 2003; 421:231-7. [PubMed: 12529635]

38. Timmons L, Fire A. Specific interference by ingested dsRNA. Nature. 1998; 395:854. [PubMed: 9804418]

39. Rual J, et al. Toward improving Caenorhabditis elegans phenome mapping with an ORFeomebased RNAi library. Genome Res. 2004; 14:2162-8. [PubMed: 15489339]

40. Mangone M, Macmenamin P, Zegar C, Piano F, Gunsalus KC. UTRome.org: a platform for 3'UTR biology in C. elegans. Nucleic Acids Res. 2008; 36:D57-62. [PubMed: 17986455]

41. Lall S, et al. A Genome-Wide Map of Conserved MicroRNA Targets in C. elegans. Current Biology. 2006; 16:460-471. [PubMed: 16458514]

42. Stark A, Brennecke J, Bushati N, Russell R, Cohen S. Animal MicroRNAs Confer Robustness to Gene Expression and Have a Significant Impact on 3'UTR Evolution. Cell. 2005; 123:1133-1146. [PubMed: 16337999]

43. Griffiths-Jones S, Saini HK, Dongen SV, Enright AJ. miRBase: tools for microRNA genomics. Nucleic Acids Research. 2007; 36:D154-D158. [PubMed: 17991681]

44. Watanabe Y, et al. Computational analysis of microRNA targets in Caenorhabditis elegans. Gene. 2006; 365:2-10. [PubMed: 16356665]

45. Johnson SM, et al. RAS is regulated by the let-7 microRNA family. Cell. 2005; 120:635-47. [PubMed: 15766527]

46. Ding XC, Slack FJ, Grosshans H. The let-7 microRNA interfaces extensively with the translation machinery to regulate cell differentiation. Cell Cycle. 2008; 7:3083-90. [PubMed: 18818519]

47. Abrahante JE, et al. The Caenorhabditis elegans hunchback-like gene lin-57/hbl-1 controls developmental time and is regulated by microRNAs. Dev Cell. 2003; 4:625-37. [PubMed: 12737799]

48. Slack FJ, et al. The lin-41 RBCC gene acts in the C. elegans heterochronic pathway between the let-7 regulatory RNA and the LIN-29 transcription factor. Mol Cell. 2000; 5:659-69. [PubMed: 10882102]

49. Lin $\mathrm{S}$, et al. The $\mathrm{C}$ elegans hunchback homolog, hbl-1, controls temporal patterning and is a probable microRNA target. Dev Cell. 2003; 4:639-50. [PubMed: 12737800]

50. Papadopoulos GL, Reczko M, Simossis VA, Sethupathy P, Hatzigeorgiou AG. The database of experimentally supported targets: a functional update of TarBase. Nucleic Acids Res. 2009; 37:D155-8. [PubMed: 18957447]

51. Xiao F, et al. miRecords: an integrated resource for microRNA-target interactions. Nucleic Acids Res. 2009; 37:D105-10. [PubMed: 18996891]

52. Stajich JE, et al. The Bioperl toolkit: Perl modules for the life sciences. Genome Res. 2002; 12:1611-8. [PubMed: 12368254]

53. Keller A, Eng J, Zhang N, Li X, Aebersold R. A uniform proteomics MS/MS analysis platform utilizing open XML file formats. Mol Syst Biol. 2005; 1:2005-0017. [PubMed: 16729052] 


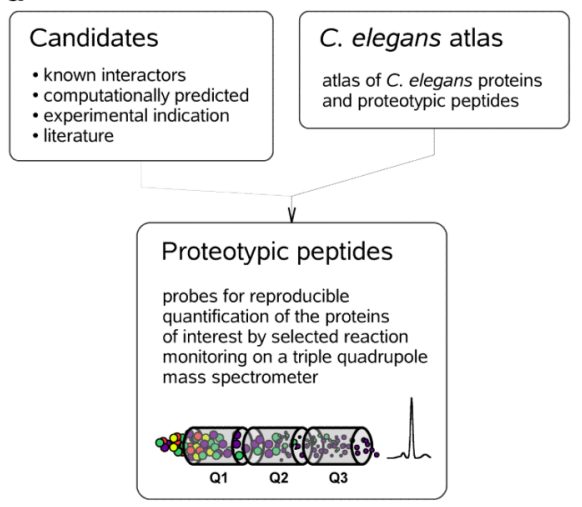

b

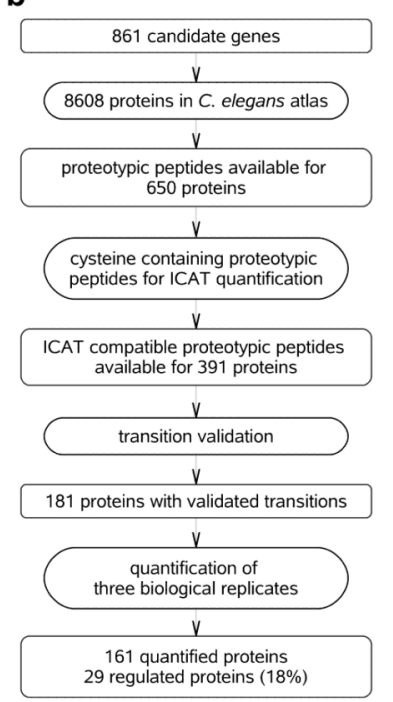

Figure 1. Strategy and workflow for targeted protein quantification

(a) General proteomic strategy and (b) workflow for the quantification of potential $C$. elegans let-7interacting genes. (a) A list of potential let-7 targets was compiled based on predictions from five different algorithms, experimental data (e.g. microarray analysis, RNAi screens, etc.) and the published literature, including known let-7 target genes. We also included control genes that we knew to be altered in let-7 mutant worms due to secondary effects (Hurschler B et al., unpublished data) and randomly chosen genes which served as "neutral controls", resulting in a final list of 861 candidate genes (Supplementary Table 1, details in "Material and Methods"). PTPs for these proteins of interest were selected from the $C$. elegans proteome atlas ${ }^{30,31}$, a recently published large $C$. elegans proteomics dataset, in which 8608 proteins, or about $40 \%$ of the proteome was identified by shotgun proteomics experiments. The selected PTPs were used as probes for reproducible quantification by SRM on a QTrap mass spectrometer operated as a triple quadrupole instrument. (b) From the initial set of 861 proteins, 650 had PTPs in the $C$. elegans proteome atlas, of which 391 had cysteine containing peptides and were quantifiable by ICAT. Validated transitions were derived for 181 proteins, of which 161 could be quantified. Of these, 29 proteins showed significant changes in abundance between wild type and let-7(n2853) mutants. 

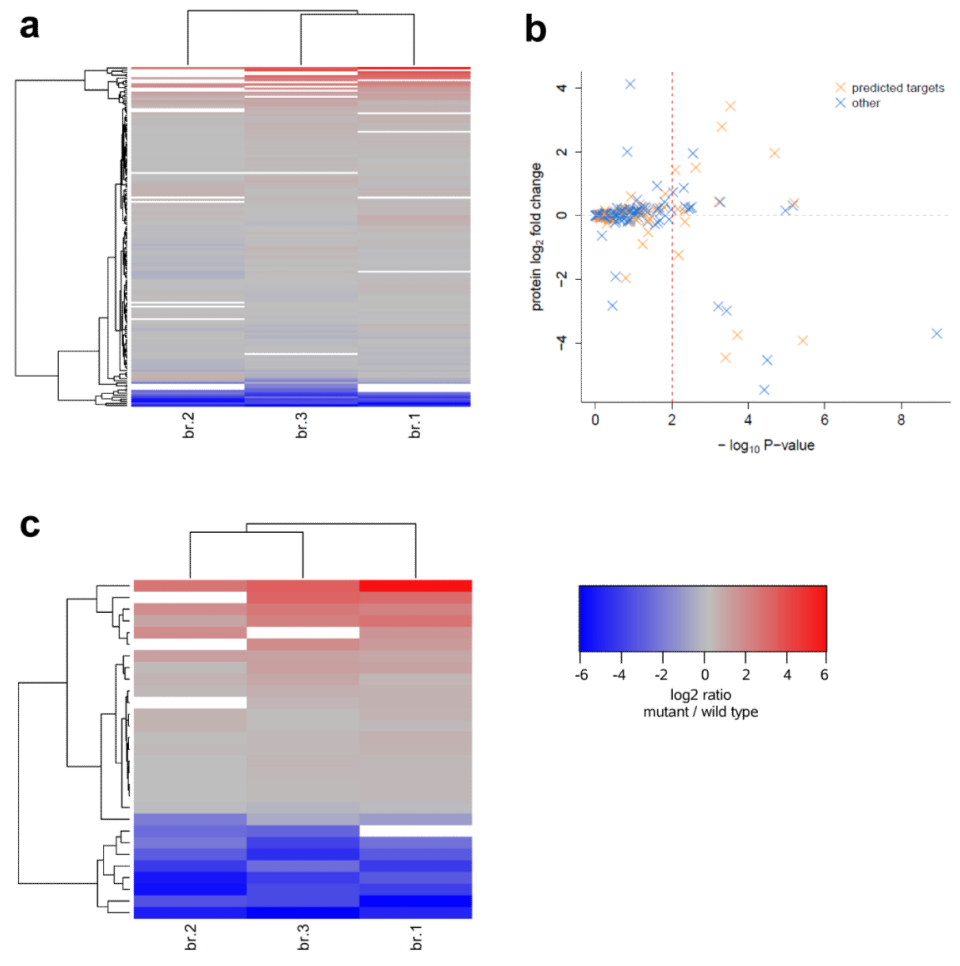

Figure 2. Identification of proteins regulated by the let-7 miRNA

(a) Heat map and hierarchical clustering of the 161 quantified proteins in three separate biological replicates (b.r.1 - b.r.3). Red and blue indicate up- and downregulated proteins in let-7(n2853) mutants, respectively (see color code). (b) Volcano plot: normalized mean log2 ratios and probability of regulation (- $\lg (P$-value $))$ of the measured proteins. Predicted let-7 targets (see "Materials and Methods") are shown in orange, all other proteins in blue. All proteins above the dotted red line (at $P=0.01$ ) were considered to be significantly regulated. (c) Heat map and hierarchical clustering of the 29 significantly regulated proteins $(P<0.01)$. 
a
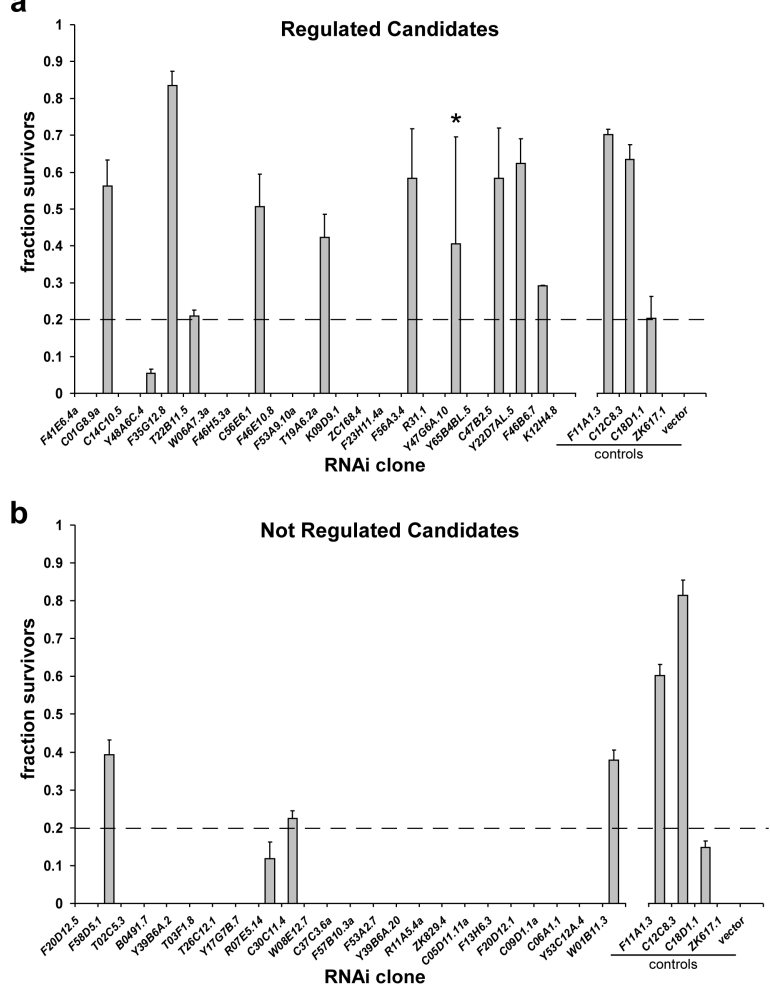

Figure 3. Genes displaying protein changes in let-7(n2853) mutants are enriched in let-7 suppressors

let-7(n2853) worms grown at $25^{\circ} \mathrm{C}$ die at the adult stage due to vulval bursting. Knockdown of some known targets has been shown to rescue this lethality to different degrees ${ }^{14}$.

(a) The 29 genes that showed protein changes were knocked-down by RNAi to determine if they suppressed the let-7(n2853) lethal phenotype. Additionally five control genes were knocked down by RNAi. The controls included two negative controls (vector and ZK617.1 (unc-22) RNAi) and three positive controls (F11A1.3 (daf-12), C12C8.3 (lin-41) and C18D1.1 (die-1) RNAi). Less than $5 \%$ of the let-7(n2853) worms treated with negative control RNAi survived as adults. Six genes could not be scored, as their RNAi inactivation led to either lethality or larval arrest. Only survival rates above $5 \%$ are shown. (b) As a control, 29 candidates that did not show a significant protein change in the let-7(n2853) mutant worms in our targeted proteomics assay were tested as in (a), including the same positive and negative controls. Again, 5/29 genes could not be scored due to lethality or larval arrest.

All the experiments were performed at $25{ }^{\circ} \mathrm{C}$ and in triplicate. The error bars depict the standard error of the mean. The candidate marked by the asterisk (*) showed suppression in two out of three replicate experiments and was regarded as positive as the average survival rate over all three replicates was above the threshold. 
a

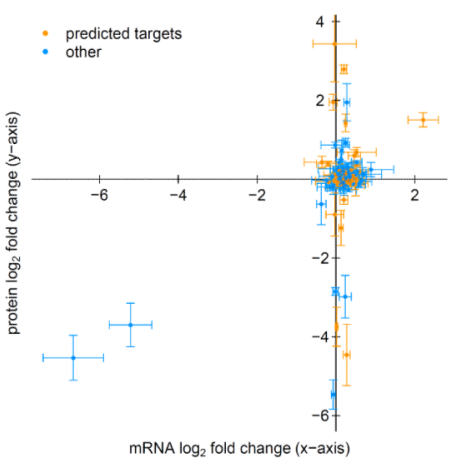

b

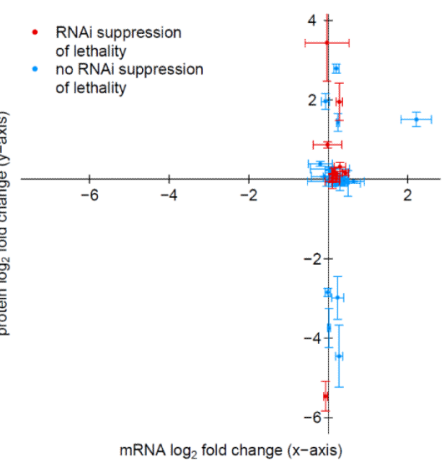

Figure 4. Comparison of let-7-dependent changes in protein and transcript levels of candidate let-7 miRNA targets

$\log 2$ fold changes at the mRNA (x-axis) and protein (y-axis) level between let-7(n2853) mutant and wild type worms for (a) all 161 proteins measured and (b) all 47 candidates scored by RNAi. (a) Proteins that are predicted let-7 targets (see "Materials and Methods") are shown in orange, all other proteins in blue. (b) Proteins that showed suppression of lethality are shown in red; proteins that did not show suppression of lethality are shown in blue. The error bars indicate the standard error of the mean over the three biological replicates. 

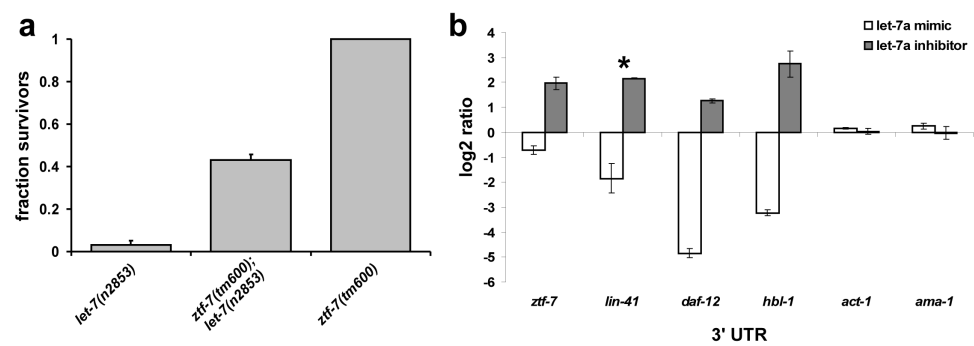

Figure 5. ZTF-7 (F46B6.7) is a bona fide let-7 target

(a) let-7(n2853) worms grown at $25^{\circ} \mathrm{C}$ die at the adult stage due to vulval bursting. Knockdown of some known targets has been shown to rescue this lethality to different degrees ${ }^{14} .3$ $\%$ of let-7(n2853) mutants are still alive 12 hours post L4. In contrast $43 \%$ of $z t f-7(t m 600)$; let-7(n2853) double mutants are alive 12 hours post L4. ztf-7(tm600) mutants did not show any lethality 12 hours post L4 and no other obvious defects. All the experiments were performed at $25^{\circ} \mathrm{C}$ and in quadruplicate. The error bars depict the standard error of the mean. (b) Relative luciferase activities for reporter constructs containing the indicated $3^{\prime}$ UTR sequences. The let-7a readouts (mimics and inhibitors) were normalized to their respective oligo controls (see "Materials and Methods" for details). The 3'UTRs of the known targets C12C8.3 (lin-41), F11A1.3 (daf-12) and F13D11.2 (hbl-1) were included as positive controls while the $3^{\prime}$ UTRs of F36A4.7 (ama-1) and T04C12.6 (act-1) were added as negative controls ${ }^{14}$. Transfections were performed in triplicate for all candidates but lin-41 (marked by asterisk $(*)$ ), which was only transfected in duplicate. The error bars depict the standard error of the mean. 


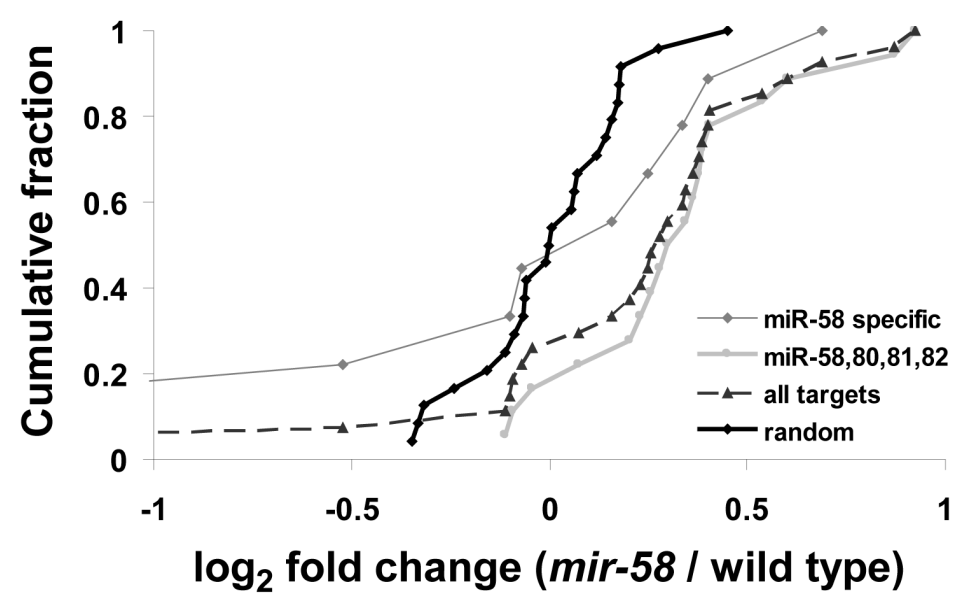

Figure 6. Predicted miR-58 targets are significantly upregulated in mir-58(n4640) mutants Comparison cumulative fraction plot of the protein response of predicted miR-58 targets (predicted by TargetScan ${ }^{18,19}$ ), also shown divided into miR-58-specific targets and targets bound by all family members and a random gene group upon mir-58 knockout. Data are shown as $\log 2$ ratios (mir-58(n4640) to wild-type). Both the overall group as well as the whole family subgroup are significantly upregulated in mir-58(n4640) mutant worms when compared to the proteins that were randomly chosen $\left(P<10^{-3}\right.$, Kolmogorov-Smirnov test). 